\title{
Fuori dalla crisi: la capacità competitiva delle imprese del distretto della meccatronica vicentina $^{1}$

\author{
Monica Plechero
}

\begin{abstract}
Obiettivo del paper: L'obiettivo del presente lavoro è di analizzare la capacità competitiva post crisi delle imprese appartenenti al settore della meccatronica vicentina, strategico oggi per l'industria 4.0 e valutare in un contesto distrettuale quali siano le caratteristiche delle imprese più performanti.

Metodologia: L'articolo si basa su dati originali raccolti nei primi mesi del 2017 su una popolazione di imprese della meccatronica. Lanalisi empirica viene svolta attraverso l'utilizzo di statistica descrittiva e lo sviluppo di un modello econometrico. Considerazioni estrapolate da alcune interviste qualitative sul territorio integrano l'analisi di natura più quantitativa.

Risultati: Il lavoro sottolinea importanti aspetti della competitiva post crisi del sistema distrettuale e suggerisce che per sostenere una capacità competitiva spendibile non è necessario essere grandi né è sufficiente investire solo negli aspetti di innovazione organizzativa e di marketing. Meccanismi virtuosi si sviluppano quando si introducono innovazioni con un alto grado di 'novelty', si formalizzano i processi di innovazione attraverso l'attività di brevettazione, si investe in risorse umane da usare nelle attività innovative e di interfaccia e si sviluppano rapporti di rete sul mercato globale.

Limiti della ricerca: La valutazione di alcune performance aziendali si basa sulla valutazione fornita dall'imprenditore stesso o da un dirigente con conoscenze approfondite dell'azienda. L'analisi empirica permette di investigare la relazione fra le variabili ma non fornisce indicazioni sulla causalità. Ricerche future potrebbero essere indirizzate ad integrare la ricerca con ulteriori indicatori statistici di natura più oggettiva e relativi agli anni subito seguenti l'indagine.

Implicazioni pratiche: L'articolo contribuisce a fornire un benchmark agli imprenditori locali fornendo indicazioni sullandamento delle imprese del sistema. Esso provvede inoltre a dare indicazioni sui fattori che sembrano sostenere al meglio la capacità competitiva post crisi.

Originalità del paper: Il lavoro si basa su dati originali che danno nuove indicazioni sui trend performativi delle imprese allinterno di un sistema ad impresa diffusa. In particolare il lavoro permette di mettere in relazione la capacità competitiva post crisi delle imprese con alcune caratteristiche delle imprese stesse, la loro attività di innovazione e la capacità di sviluppare rapporti con il sistema locale e con imprese e organizzazioni a livello internazionale.

1 Il presente articolo è frutto di un lavoro inizialmente sviluppato dallautrice presso il Dipartimento di Management, Università di Venezia e facente parte del progetto di ricerca: "Alla ricerca di nuovi modelli di business: il riposizionamento delle imprese italiane nel contesto della concorrenza globale" (anno accademico 2016/2017).
\end{abstract}


sinergie

Vol. 36, N. 107, 2018

Parole chiave: meccatronica; distretto industriale; capacità competitiva; innovazione; post crisi

\section{Out of the Crisis: Firms' competitive capacity in the Vicenza Mechatronics District}

Purpose of the paper: The paper aims at analyzing the post crisis competitive capacity of firms belonging to Vicenza mechatronics industry, that nowadays represents a strategic sector for pursuing Industry 4.0 objectives. The study wants also to disclosure which firms' characteristics in the local system allow to reach a certain degree of competitive performance.

Methodology: The study is based on original data collected on a population of mechatronics firms in the first months of 2017. The analysis, integrated also with insights from some qualitative interviews conducted in loco, is mainly based on descriptive statistics and econometric analysis.

Findings: The study underlines important aspects of mechatronics industrial district's competitive capacity, suggesting that to sustain valuable performances neither is necessary to reach a certain size nor sufficient to invest only in commercial and organizational innovation. Findings suggest that virtuous mechanisms are triggered when 1. innovation with high degree of novelty are introduced, and 2. formalized by patents activities, 3. there is a certain presence of human resources to dedicate to innovation and that can act as interface with the outside world, and 4. network relations at global level are developed.

Research limits: Assessments of firms' performance are based on entrepreneurs or expert managers' evaluations. Causality among variables cannot be assessed. Future research could integrate the research results with more objective statistical data related to the years following this first analysis.

Practical implications: The paper provides local entrepreneurs with a useful benchmark and new knowledge about trends in the local system. It gives also some guidelines about which factors may best sustain post crisis competitive positions.

Originality of the paper: This study is based on original data which provide new insight on firms' trend performances within an industrial district. In particular, the research allows to relate firms' post crisis competitive position with firms' characteristics, firms' innovation activities and firms' capability to develop network relation both with locals well as international firms and organizations.

Key words: mechatronics; industrial district; competitive capacity; innovation; post crisis

\section{Introduzione}

La transizione attuale relativa alla globalizzazione e rivoluzione digitale se da una parte riduce la forza dei modelli competitivi ispirati al passato (in particolare del capitalismo distrettuale), dallaltra apre anche nuove possibilità a quelle aziende della manifattura italiana che hanno saputo stare al passo con la modernità in cui si richiede da una parte una frattura con alcuni aspetti della tradizione e dall'altra una continua esplorazione del nuovo (Rullani, 2010). Superando i problemi e le incertezze legate alla crisi 
2008-2014, alcune imprese del distretto meccatronico vicentino, oggetto di tale studio, hanno saputo trovare modelli di business vincenti adottando una visione strategica delle potenzialità di generazione del valore che sono presenti o latenti.

Il presente articolo, attraverso l'analisi di dati originali, raccolti nel 2017, vuole contribuire a mettere in evidenza le caratteristiche delle imprese che hanno dimostrato di aver superato la crisi con una certa capacità competitiva non solo nei confronti della concorrenza locale e nazionale ma anche estera, seguendo percorsi di innovazione ma anche sfruttando i legami che si sviluppano in rete. Le domande di ricerca a cui il presente lavoro vuole rispondere sono: Quale è il livello di capacità competitiva raggiunto dalle imprese nel periodo post crisi? Quali fattori e performances sono associati ad un certo grado di capacità competitiva? La sezione 2 di inquadramento teorico dopo aver fornito una panoramica sulla situazione dei distretti mette in luce i fattori di contesto e di impresa che in letteratura sono solitamente associati al raggiungimento di buone performances competitive da parte delle imprese. La sezione 3 descrive come sono stati raccolti e analizzati i dati mentre la sezione 4 presenta e discute i risultati empirici atti ad evidenziare i fattori associati ad un raggiungimento di vari gradi di capacità competitiva da parte delle imprese del distretto della meccatronica vicentina. La sezione 5 conclude fornendo alcune raccomandazioni di policy.

\section{Inquadramento teorico}

\section{La capacità competitiva post crisi dei distretti}

A partire dagli anni 2000 il capitalismo distrettuale ha iniziato ad andare in crisi. Per diversi studiosi, la causa è il modello sociale ed economico stesso, su cui il capitalismo distrettuale si basa, che è stato incapace di far fronte prontamente ai percorsi sempre più sofisticati di globalizzazione seguendo giuste traiettorie tecnologiche. Il modello distrettuale che sfruttando le economie di agglomerazione e l'apprendimento collettivo basato sul saper fare ha permesso in passato lo sviluppo di produzioni locali flessibili, specializzate e di valore per le piccole e medie imprese (Becattini, 1998), sembra non funzionare più come prima. Sebbene all'inizio dell'ultima crisi finanziaria diversi sono stati i contributi dei distrettualisti che hanno cercato di mettere in evidenza scenari possibili per i distretti italiani (Rabellotti et al., 2009; Dei Ottati, 2009; Rullani, 2010), i contributi sulla competitività distrettuale sono andati via via negli ultimi anni scemando, perdendo il distretto l'originale appealing che aveva avuto fra gli studiosi durante gli anni d'oro ' 80 e ' 90 . In realtà l'idea che sembra essere prevalsa è che nei sistemi distrettuali esiste oggi una certa capacità competitiva eterogenea più che di sistema (Boschma e ter Wal, 2007; Belussi e Sedita, 2009). Da una parte si trovano quelle imprese - soprattutto di piccola dimensione - incapaci di rinnovarsi, dall'altra isolate ma vincenti iniziative da parte di imprese leader, spesso di media dimensione o appartenenti a gruppi di impresa capaci di trovare - senza mantenere legami stringenti con il territorio - un proprio originale percorso di crescita. meccatronica vicentina 
sinergie Vol. 36, N. 107, 2018

Lultima crisi finanziaria ha dato poi un'ulteriore scossa a molte imprese che caratterizzano la manifattura italiana. In molti contesti distrettuali italiani sono emerse le difficoltà da parte di molte imprese di inserirsi in reti cognitive estese o di costruire conoscenze locali rigenerative in grado di mettere in moto il ri-uso specializzato della conoscenza adeguato ad affrontare da una parte i rischi finanziari della crisi e dallaltra una concorrenza sempre più globale (Rullani, 2010; 2015).

I recenti rapporti sui sistemi distrettuali italiani (Intesa San Paolo, 2015; Unioncamere, 2014; 2015) mostrano tuttavia una certa ripresa da parte di alcuni distretti. Secondo losservatorio dei distretti (Unioncamere, $2014 ; 2015)$ la meccanica e meccatronica italiana, fra cui quella vicentina, è stata trainante nell'export complessivo del paese mostrando buoni indici di fatturato, margini e redditività. In questo quadro sembra che siano state soprattutto le medie imprese quelle che sono state capaci di mantenere una certa solidità dal punto di vista competitivo (Coltorti, 2015; MediobancaUnioncamere, 2015). Ad ogni modo quello che dai rapporti risulta chiaro è che per uscire dalla crisi e fare esperienza positiva della modernità in cui la globalizzazione e la digitalizzazione diventano processi inarrestabili, è necessario un rinnovamento dei modelli di business. Per tale motivo analizzare quali siano in generale i principali fattori che possono sostenere la capacità competitiva in un sistema a impresa diffusa come quello della meccatronica vicentina può tornare utile per valutare se possono essere individuati comportamenti premiali comuni fra le imprese o se una reale capacità competitiva resta in realtà prerogativa di poche iniziative isolate che forniscono una differenza solo apparente fra un sistema distrettuale e un altro.

\section{I fattori che sostengono la capacità competitiva}

Diversi studiosi di distretti e sistemi locali sostengono che l'analisi del tipo di governance locale, così come delle politiche applicate a livello di sistema, sia fondamentale per comprendere l'evoluzione della capacità competitiva dei sistemi a impresa diffusa (Ricciardi, 2013; Bailey et al. 2010). Visto l'attuale eterogeneità dei comportamenti registrati nei sistemi locali, particolare attenzione va però data anche alla specifica capacità competitiva del settore e delle sue diverse specializzazioni, così come delle singole imprese che ne fanno parte. Esiste un legame importante fra performances soprattutto di tipo innovativo e strutture, conoscenze di base e dinamiche specifiche di settore (Malerba, 2005; Pavitt, 1984). Il settore della meccatronica, oggetto di questo studio, è un settore che combinando conoscenze di meccanica, informatica ed elettronica risulta non inquadrabile in una specifica classifica merceologica. Esso raggruppa nel suo insieme aziende produttrici di macchine strumentali al servizio dell'industria ma anche dell'agricoltura, aziende focalizzate sulle attività di automazione così come aziende dedicate alla produzione di motori, pompe elettriche e strumentazione di precisione o altri prodotti di componentistica elettronica rivolte a specifiche attività manifatturiere che vanno dal tessile alla produzione alimentare. Per tale motivo le performance competitive della meccatronica potrebbero seguire dinamiche non tanto di settore nel suo insieme, ma dei suoi sotto-settori di specializzazione, che possono rispondere a nuclei di know how diversi (Bellandi et al., 2018). 
Nella specifica letteratura distrettuale si è poi spesso evidenziato come - grazie alla maggiore disponibilità di risorse interne e di capitale - le aziende medie siano in grado di presenziare in modo competitivo i mercati internazionali più delle piccole, assumendo spesso un ruolo di leadership per quest'ultime (Chiarvesio et al., 2010; Coltorti et al., 2012; Rabbellotti et al., 2009; Morrison, 2008).

La letteratura internazionale, soprattutto appartenente al filone classico della resource based view (Penrose, 1959; Teece, 1980; Wernefelt, 1984), associa poi le performances competitive delle imprese più che alle caratteristiche strutturali come la dimensione d'impresa o il settore di appartenenza, al possesso in azienda di specifiche risorse e competenze. Molti contributi sui sistemi distrettuali vanno oggi in questa direzione, evidenziando l'eterogeneità delle capacità competitive che spesso esistono all'interno dello stesso distretto (Boschma e ter Wal, 2007; Belussi e Sedita, 2009) e la necessità di sviluppo di tali competenze per sostenere la resilienza del sistema locale. Non si tratta solo di risorse e competenze di natura tecnologica legate agli aspetti di innovazione, e ampiamente discusse in letteratura (Antonelli, 1995; Bell e Pavitt, 1993), ma anche di natura più cognitiva o immateriale (Sedita et al., 2017). L'innovazione che sostiene la capacità competitiva delle imprese oggi assume caratteristiche più complesse e aperte, in cui all'aspetto tecnologico si affianca ad esempio l'aspetto gestionale, necessario a rendere più produttiva la conoscenza prodotta e a valorizzare l'integrazione fra competenze e conoscenze nuove e tradizionali (Varaldo, 2014). In base alla definizione tratta dal manuale di Oslo (OECD, 2005) l'innovazione deve essere intesa come attività che riguarda non solo l'innovazione di prodotto cioè l'introduzione di un nuovo o considerevolmente migliorato bene o servizio, ma anche l'innovazione di processo, organizzativa e di marketing. Queste ultime due (innovazioni organizzative e di marketing) sono innovazioni spesso funzionali al core innovativo dell'azienda, sia esso relativo alla produzione in quanto tale o al processo con cui un bene o servizio viene consegnato o customizzato (Plechero e Rullani, 2007). Diversi studiosi evidenziano la relazione positiva e spesso di causalità circolare che esiste fra le varie attività di innovazione e la possibilità di raggiungere migliori performance economiche e capacità competitive (es. Cainelli et al., 2006; Hall et al., 2009).

$\mathrm{Al}$ di là di risorse dedicate alle attività di ricerca e sviluppo e alla messa a disposizione di un proprio laboratorio interno e della capacità di formalizzare la propria innovazione attraverso l'attività di brevettazione (Gustavsson et al., 1999; Chiarvesio et al., 2010), la letteratura sottolinea che per sviluppare competitività e capacità strategica sul lungo periodo è necessario investire in risorse umane. Un'elevata qualità del capitale umano è infatti considerata una fra le più classiche determinanti del vantaggio competitivo d'impresa (Itami, 1987). La capacità tradizionale del sistema distrettuale si basa sul saper fare dell'imprenditore e dei suoi collaboratori, su conoscenza tacita ed esperienza acquisita, sull'apprendimento sul campo dei dipendenti e la formazione in fabbrica delle maestranze. Con l'avanzamento tecnologico e la digitalizzazione, gli aspetti tecnici e ingegneristici diventano però talmente sofisticati che risulta sempre
Monica Plechero

capacità competitiva delle imprese del distretto della meccatronica vicentina 
sinergie Vol. 36, N. 107, 2018

più necessario avere in azienda chi è in grado di governare le attività $\mathrm{o} i$ processi più 'analitici'. Ciò richiede l'impiego di personale qualificato nei processi innovativi che abbia un background di studi capace di fornire conoscenze teoriche oltre che tecniche. Vari studi dimostrano che ad un buon livello di capitale umano nelle piccole e medie imprese (anche in termini di grado di istruzione) è associata una maggiore crescita aziendale e migliori performance competitive (Colombo e Grilli, 2005; Farace e Mazzotta, 2015). Questo capitale alimenta la cosiddetta absortive capacity dell'impresa (Cohen e Levinthal, 1990), che altro non è che la capacità di interfaccia dellazienda di assimilare, riconoscere e saper valorizzare le informazioni e le conoscenze che dall'esterno dell'azienda si propagano allinterno e viceversa.

Negli anni doro dei distretti il capitale territoriale locale (Barzotto et al., 2016) - che si è formato attraverso i rapporti di sistema - ha permesso lo sviluppo di fattori di produzione altamente competitivi permettendo sia la moltiplicazione delle specializzazioni in loco, sia la generazione di importanti economie di varietà costruite su base comune. La collaborazione con le altre aziende del territorio ha messo in moto meccanismi di supporto e apprendimento collettivo che hanno fatto in modo che si potessero raggiungere le giuste economie di scala garantendo al contempo anche produzioni di qualità e di nicchia (Marshall, 1920; Maskell e Malberg, 1999)

A partire dagli anni 2000, proprio quando il capitalismo distrettuale stava affrontando le prime crisi, gli studiosi di geografia economica sono stati tuttavia fra i primi a sottolineare come i vantaggi competitivi dellagglomerazione geografica siano da soli insufficienti per affrontare i processi di globalizzazione in unottica di innovazione. Inserirsi in reti che catturano valore sia locale che globale diventa per le imprese la nuova chiave per sviluppare capacità competitive strategiche (Bathelt et al., 2004). Per far fronte alla concorrenza globale si richiede oggi capacità di partecipare a reti transnazionali attraverso lo sviluppo di collaborazioni nellattività produttiva e innovativa con imprese e organizzazioni estere (Belussi e Sedita, 2012; Rullani, 2004; De Marchi et al., 2018) sia per immettere nel territorio nuove idee, ma anche per moltiplicare nel modo più efficace possibile le proprie potenzialità garantite anche da semplici innovazioni d'uso (Castellani et al., 2017). La capacità di relazionarsi con contesti esteri non è sempre facile. Se da una parte è necessario una minima prossimità cognitiva con gli attori globali attraverso lo sviluppo di unadeguata absortive capacity da parte dell'azienda, dall'altra è necessario possedere e saper governare le giuste tecnologie digitali per sviluppare rapporti a distanza, comprendere rapidamente le esigenze del cliente globale anticipandole (Castellani et al., 2017).

\section{Metodologia}

Il presente studio che intende valutare la capacità competitiva delle imprese del sistema della meccatronica vicentina attraverso l'analisi dei fattori descritti nell'inquadramento teorico, si basa su dati originali raccolti attraverso unindagine messa on line con il software Qualtrics 
durante febbraio-marzo 2017. L'indagine - che ha avuto come principale scopo indagare le varie attività di internazionalizzazione delle imprese e il loro legame con gli aspetti di innovazione - è stata sviluppata sulla base di domande originali, anche se fortemente ispirate da alcune contenute nella community innovation survey (CIS) e in altri indagini effettuate per progetti con tematiche simili, ma testate su paesi e settori diversi ${ }^{2}$. L'indagine ha avuto come target le aziende della meccatronica della provincia di Vicenza (aventi un minimo di 5 dipendenti). Il vicentino rappresenta l'area in cui vi è la maggior concentrazione di aziende venete in tale settore e costituisce il cuore del distretto della meccatronica. Poiché il settore non è riconducibile di per sé ad una specifica classificazione merceologica le aziende sono state individuate guardando ad una serie di codici $^{3}$ ATECO che in un precedente rapporto (Universitas Mercatorum, 2013) sono stati ricondotti a tre principali attività con maggiore vocazione meccatronica nell'area: macchine per l'industria, macchine ed apparecchi elettrici ed elettronici e automazione. Dopo aver individuato la popolazione sottostante tali criteri, le aziende sono state controllate una per una attraverso informazioni raccolte in internet. $\grave{E}$ stato possibile in questo modo eliminare quelle aziende con evidente attività non collegata alla meccatronica, con sede operativa fuori provincia, aziende in fase di liquidazione e aziende prive di sito on line o indirizzo email necessario per il contatto. In totale il questionario è stato inviato ad una popolazione di 460 imprese. Il questionario conteneva una serie di 29 domande strutturate relative a: caratteristiche dell'azienda (dimensione, appartenenza a gruppi di impresa, età e sotto-settore), capacità competitiva post crisi, profilo dei dipendenti, attività e capacità innovativa, attività e strategie di internazionalizzazione (es. export, investimenti diretti all'estero), rapporti di rete (con chi e con che estensione geografica) e rapporti con il territorio locale (es. ruolo di altre organizzazioni locali nel sostenere la capacità competitiva e l'internazionalizzazione $)^{4}$. Un primo questionario pilot è stato inviato a 3 imprese e 6 esperti in varie materie (meccatronica, internazionalizzazione, innovazione e sistemi distrettuali). Dopo aver ricevuto i vari feedback ed effettuati alcuni minimi riadattamenti, il questionario è stato inviato all'intera popolazione fra febbraio e marzo 2017. Il questionario è stato indirizzato specificatamente all'imprenditore o amministratore delegato o, quando questi soggetti non erano raggiungibili, a personale con conoscenze approfondite dell'azienda e con l'autorizzazione da parte dellorganizzazione alla compilazione. In molti casi le aziende sono state sollecitate telefonicamente a rispondere. Il tasso di risposta è del $18,7 \%^{5}$. Secondo il Person Chi Squared test dal punto di vista della

2 Si vedano a proposito i lavori relativi di Plechero e Chaminade (2013); Chaminade e Plechero (2015) e Martin et al. (2018).

3 Per l'individuazione delle aziende, svolta attraverso il database AIDA e integrato con i dati della camera di commercio (CCIAA) sono state estratte tutte le aziende della provincia appartenenti ai codici ATECO 2007: C262, C2711, C28, C332001, C332002, C332003, C332006.

4 Il questionario completo è disponibile da parte dellautore su richiesta.

5 Tale popolazione esclude alcune aziende ad evidente attività meccatronica dell'area ma che appartenendo a codici diversi da quelli individuati non sono state incluse nel database.
Monica Plechero

capacità competitiva delle imprese del distretto della meccatronica vicentina 
sinergie Vol. 36, N. 107, 2018

dimensione aziendale le 86 aziende che hanno risposto al questionario rappresentano in modo significativo le imprese della popolazione vicentina individuata. L'indagine è stata poi ulteriormente integrata con un'intervista a due funzionari dellassociazione imprenditoriale vicentina e 6 interviste fatte alle imprese locali che si sono particolarmente distinte per le loro performance dal punto di vista dei percorsi di innovazione e internazionalizzazione intrapresi.

Per il presente articolo viene utilizzata inizialmente un'analisi di statistica descrittiva relativa agli aspetti di competitività delle aziende. Viene poi presentato il modello econometrico basato su una generalized ordered logit per mettere in relazione il grado di capacità competitiva dellazienda con alcune caratteristiche, risorse e competenze delle imprese desunte dall'indagine e discusse nella sezione relativa allinquadramento teorico. I grafici finali forniscono un quadro delle performance economiche delle imprese con diverso grado di competitività.

\section{L’analisi empirica}

Per comprendere lattuale capacità competitiva delle imprese, alle aziende partecipanti all'indagine è stato chiesto di indicare la propria posizione competitiva post crisi (2008-2014), scegliendo tra un numero limitato di opzioni (Tab. 1).

Tab. 1: Posizione competitiva post crisi

\begin{tabular}{|l|c|c|c|}
\hline Posizione competitiva post crisi (2008-2014): & Frequenza & Percentuale & $\begin{array}{l}\text { Percentuale } \\
\text { Cumulata }\end{array}$ \\
\hline Più deboli (1) & 7 & 8,14 & 8,14 \\
\hline Incapaci di rispondere ai cambiamenti di mercato (2) & 2 & 2,33 & 10,47 \\
\hline Uguali a prima (3) & 10 & 11,63 & 22,09 \\
\hline $\begin{array}{l}\text { Più strategici e selettivi ma non più forti della } \\
\text { concorrenza (4) }\end{array}$ & 36 & 41,86 & 63,95 \\
\hline Più forti rispetto alla concorrenza locale (5) & 7 & 8,14 & 72,09 \\
\hline Più forti rispetto alla concorrenza nazionale (6) & 11 & 12,79 & 84,88 \\
\hline Più forti rispetto alla concorrenza internazionale (7) & 13 & 15,12 & 100 \\
\hline Totale & 86 & 100 & \\
\hline
\end{tabular}

Fonte: Elaborazioni su dati raccolti

Come si può notare dai dati estrapolati la maggior parte delle aziende che hanno risposto all'indagine hanno indicato che la loro posizione post crisi è migliore rispetto al periodo 2008-2014. Sebbene la popolazione selezionata è già al netto di imprese in corso di liquidazione o con situazione fallimentare, è necessario tener conto di un possibile bias di risposta dovuto al fatto che aziende con situazioni più favorevoli tendono naturalmente a rispondere alle indagini più delle aziende con situazioni negative. La maggior parte delle aziende $(41,86 \%)$ non indica comunque una reale capacità competitiva, ma solo una maggior capacità strategica. Esse infatti hanno risposto che si sentono più strategiche e selettive dopo la crisi ma non più forti della concorrenza. Un buon numero di 
aziende indica di possedere una certa capacità competitiva non solo nei confronti della concorrenza locale $(8,14 \%)$ e nazionale $(12,79 \%)$, ma anche internazionale $(15,12 \%)$. Per valutare se esiste una relazione fra capacità competitiva espressa dalle imprese e caratteristiche delle imprese discusse nella sezione di inquadramento teorico, viene effettuata una semplice analisi econometrica che rende opportuno (visto il numero limitato di imprese) il raggruppamento di alcune categorie nominali di scelta indicate in tabella 1. Tale raggruppamento permette di arrivare ad ottenere una variabile ordinale con valore 0 per indicare una posizione competitiva post crisi dell'impresa più debole o stabile (categorie 1,2 e 3 della tab. 1), con valore 1 per indicare una posizione invece più strategica e selettiva ma non più forte di quella della concorrenza (categoria 4 della tab. 1 ) e con valore 2 per indicare una posizione competitiva più forte (5,6 e 7 della tab. 1). Il modello presentato in tabella 2 , come anticipato nella precedente sezione, è una generalized ordered logit ${ }^{6}$ che permette di valutare la relazione tra la variabile dipendente appena costruita e il profilo delle imprese associato ai cambiamenti delle loro capacità competitive dopo la crisi. In particolare il modello permette di mettere in luce nella colonna A come alcune variabili indipendenti si relazionino al posizionamento competitivo delle imprese con valore 1 e 2 rispetto alle imprese con valore 0 e nella colonna B come tali variabili si relazionino con il posizionamento competitivo delle imprese con valore 2 rispetto alle imprese sia con valore 0 che con valore 1.

Come variabili indipendenti sono state considerate alcune variabili associate alle risorse e capacità innovative delle imprese, alla rilevanza che assumono le altre imprese locali nel sostenere la capacità competitiva dell'impresa e l'esistenza di legami di natura internazionale, in particolare di collaborazioni internazionali per attività di produzione o innovazione in cui vi sia stato un reale scambio di conoscenze. Le variabili di controllo sono relative ad alcune caratteristiche strutturali quali la dimensione dell'impresa $^{7}$ e il sotto-settore principale di appartenenza in cui viene distinto il settore delle macchine per l'industria - che rimane prevalente dagli altri settori.

La descrizione delle variabili, così come la statistica descrittiva e relativa tabella di correlazione vengono riportate in appendice.

Come si desume dal modello, il possedere una certa dimensione (almeno media) non sembra essere un fattore fondamentale né per il raggiungimento da parte dellazienda di una migliore capacità strategica, né per riuscire a raggiungere un miglioramento delle capacità competitive. Rilevante risulta invece essere il sotto-settore di appartenenza

6 Il modello viene solitamente utilizzato al posto di un modello di logit ordinario quando l'assunzione di proporzionalità degli odds (POA) viene violata. Contrariamente alla ordered logit, e in assenza di POA, la generalized ordered logit permette di stimare coefficienti diversi per diverse categorie. Per un approfondimento sul modello si veda Williams (2006).

7 Si distingue tra aziende di piccola dimensione da una parte ( $<50$ dipendenti) e medie e grandi dall'altra Tuttavia solo lo $3,48 \%$ della popolazione è rappresentato da aziende che superano i 250 dipendenti, mentre le aziende di media dimensione rappresentano il $18,61 \%$ di tutta la popolazione rispondente. Ciò ricalca alquanto realisticamente la tipica stratificazione dimensionale delle imprese distrettuali. 
sinergie italian journal of management Vol. 36, N. 107, 2018 (l'appartenenza alla categoria di produttori di macchine per l'industria rispetto agli altri sotto-settori in cui il lavoro preponderante è relativo allattività di automazione, produzione macchine utensili o addirittura, come nel caso di alcune imprese, la produzione di macchine agricole). In realtà, nel campione esiste un certo numero di imprese specializzate in produzioni di macchine industriali (come le macchine che servono l'industria conciaria), che stanno mantenendo buone performances sui mercati. Uno degli imprenditori delle aziende intervistate del settore, sottolinea però che in questo momento sebbene ci sia una buona tenuta delle aziende locali sul mercato nazionale, a livello internazionale si inizia a sentire la concorrenza che avanza da parte di aziende in India, Brasile, Cina e Vietnam. Sono concorrenti che, sebbene offrano normalmente produzioni di qualità inferiore, rappresentano comunque una minaccia sempre più imponente per le aziende locali.

\section{Tab. 2: Generalized ordered logit}

Variabile dipendente: posizione competitiva dellimpresa: 0 posizione competitiva post crisi debole o stabile; 1 posizione più strategica e selettiva (ma non più forte della concorrenza); 2 posizione più competitiva

\begin{tabular}{|c|c|c|c|}
\hline \multicolumn{2}{|l|}{$\mathrm{A}-\mathrm{Da}(0) \mathrm{a}(1 \circ 2)$} & \multicolumn{2}{|l|}{$\mathrm{B}-\mathrm{Da}(0$ o 1$)$ a 2} \\
\hline Dimensione & $-0,116$ & Dimensione & 0,977 \\
\hline & {$[1,013]$} & & {$[0,844]$} \\
\hline \multirow[t]{2}{*}{ Settore macchine per l'industria } & $1,470^{\star *}$ & Settore macchine per l'industria & $3,881^{\star * *}$ \\
\hline & {$[0,716]$} & & {$[1,145]$} \\
\hline \multirow[t]{2}{*}{ Dipartimento R\&S } & $-0,532$ & Dipartimento R\&S & 1,102 \\
\hline & {$[0,847]$} & & {$[0,783]$} \\
\hline \multirow[t]{2}{*}{$\begin{array}{l}\text { Background } \\
\text { ingegneristici }\end{array}$ HR studi tecnici/ } & 0,831 & \begin{tabular}{|l}
$\begin{array}{l}\text { Background HR studi tecnici/ } \\
\text { ingegneristici }\end{array}$ \\
\end{tabular} & $2,548^{* *}$ \\
\hline & {$[0,810]$} & & {$[1,217]$} \\
\hline \multirow[t]{2}{*}{ Brevetti } & 1,775 & Brevetti & $-0,277$ \\
\hline & {$[1,371]$} & & {$[0,814]$} \\
\hline \multirow[t]{2}{*}{ Innovazione all'avanguardia } & 0,059 & Innovazione all'avanguardia & $2,586^{* *}$ \\
\hline & {$[1,246]$} & & {$[1,131]$} \\
\hline \multirow[t]{2}{*}{$\begin{array}{llll}\begin{array}{l}\text { Innovazioni } \\
\text { organizzative }\end{array} & \text { di marketing } \quad \text { e/o } \\
\end{array}$} & $1,585^{\star *}$ & $\begin{array}{|llll|}\begin{array}{l}\text { Innovazioni } \\
\text { organizzative }\end{array} & \text { marketing } \quad \mathrm{e} / \mathrm{o} \\
\end{array}$ & 0,671 \\
\hline & {$[0,800]$} & & {$[0,695]$} \\
\hline \multirow[t]{2}{*}{ Rilevanza sistema di imprese locali } & 1,329 & Rilevanza sistema di imprese locali & $3,874^{* * *}$ \\
\hline & {$[0,840]$} & & {$[1,077]$} \\
\hline \multirow[t]{2}{*}{ Collaborazioni internazionali } & $-2,108$ & Collaborazioni internazionali & $1,975^{\star *}$ \\
\hline & {$[1,532]$} & & {$[0,966]$} \\
\hline \multirow[t]{2}{*}{ Costante } & $-0,799$ & Costante & $-7,849^{* * *}$ \\
\hline & {$[0,814]$} & & {$[2,121]$} \\
\hline $\mathrm{N}$ & 84 & & 84 \\
\hline 11 & $-59,758$ & & $-59,758$ \\
\hline chi2 & 58,722 & & 58,722 \\
\hline $\mathrm{p}$ & 0,000 & & 0,000 \\
\hline $\mathrm{R}^{2}$ & 0,3295 & & 0,3295 \\
\hline
\end{tabular}

Standard error in parentesi; ${ }^{\star *} \mathrm{P}$ value significativo al $1 \%$; ${ }^{\star *} \mathrm{P}$ value significativo al $5 \%$. Due aziende avendo un missing value su due variabili non sono state incluse nell'analisi ecometrica.

Fonte: elaborazioni proprie sui dati del questionario. 
Ciò che distingue poi le aziende in possesso almeno di capacità strategiche aumentate rispetto a quelle che dopo la crisi hanno mantenuto o posizioni stabili o si sono addirittura indebolite risulta l'aver sviluppato negli ultimi tre anni (2014-2016) innovazioni di tipo organizzativo o di marketing. Si è trattato, ad esempio, di implementazioni di un nuovo metodo organizzativo nelle pratiche commerciali dell'azienda o nel luogo di lavoro o nelle relazioni esterne così come un riposizionamento di mercato o nuove promozioni relative al prodotto. Ė evidente quindi che in diversi casi la crisi ha spinto una ristrutturazione e riorganizzazione aziendale e commerciale che ha portato allo sviluppo da parte delle imprese di comportamenti più selettivi ed efficienti anche se non sufficienti da soli a raggiungere una buona capacità competitiva. Analizzando specificatamente la colonna $\mathrm{B}$ della tabella 1, che mette in evidenza la differenza fra aziende che dopo la crisi non si sentono più competitive rispetto ai concorrenti (anche se in alcuni casi hanno migliorato le loro capacità strategiche) da quelle che si sentono invece più forti dei concorrenti (siano essi locali, nazionali o internazionali) si capisce chiaramente che uno dei requisiti minimi per sviluppare capacità competitiva è il possesso in azienda di risorse umane con un certo tasso di scolarizzazione da dedicare alle attività di innovazione. Si tratta soprattutto di personale con studi tecnici specializzati o con studi ingegneristici in grado di far fronte a conoscenze sempre più complesse $\mathrm{e}$ che necessitano anche un approccio teorico al problema, la comprensione e l'analisi di dati oltre che una certa capacità di governare i processi di digitalizzazione. Ciò è confermato da tutte le aziende intervistate sia nel settore dell'automazione che delle macchine per l'industria. Una delle aziende più performanti - attraverso la voce del proprio responsabile tecnico - sottolinea la trasformazione e il successo della loro produzione verso aspetti sempre più digitali. Nella trasformazione risulta chiaro come sia importante governare in modo completo tali processi anche attraverso il dominio di vari tipi di conoscenze. 'Quindi questo oggetto fatto di ferro e plastica sta diventando un oggetto fatto di elettronica montato su una scheda con un software che gira... si tratta di tenere in piedi sia i (vecchi) linguaggi che quelli che arriveranno, si tratta di una continua integrazione di competenze di mentalità, di metodologia'.

Risulta poi significativo aver fatto negli ultimi 3 anni innovazioni all'avanguardia, ossia essere fra i primi o pochissimi a livello mondiale ad aver introdotto quel tipo di innovazione che può anche non essere radicale ma che fornisce senz'altro economie di varietà importanti e di customizzazione soprattutto sotto il profilo della produzione e del processo produttivo. Importante è poi l'aver mantenuto legami positivi con il sistema di imprese locali e aver sviluppato collaborazioni internazionali in cui ci sia stato un vero e proprio scambio di conoscenze su aspetti di produzione ma anche su attività legate agli aspetti di innovazione. Lamministratore delegato di una delle aziende intervistate specializzata nei processi di automazione che da poco ha iniziato a sviluppare anche una presenza in Cina ha ad esempio spiegato come per lo sviluppo di nuove app sia stato fondamentale affiancare un loro giovane dipendente scolarizzato e impegnato nelle attività di R\&S con un dipendente dello stesso livello in una azienda cliente operante in Cina. Il loro comune impegno nello
Monica Plechero

capacità competitiva delle imprese del distretto della 
sinergie Vol. 36, N. 107, 2018

sviluppo di investimenti all'estero non solo di produzione ma anche di R\&S sta aiutando l'azienda ad esportare con successo il proprio prodotto nel mercato cinese. Il modello fornisce alcune prime riflessioni importanti fra la relazione tra fattori che spingono la capacità competitiva delle imprese e la competitività post crisi delle aziende del tessuto locale.

La tabella 3, che confronta sui fattori analizzati le aziende con diverso grado di capacità competitiva (da una parte aziende che si sentono più forti della concorrenza locale e nazionale e dall'altra aziende che si sentono più forti della concorrenza internazionale) fornisce altri dettagli importanti. Dai dati emerge che un'alta percentuale di aziende con capacità competitive sui mercati internazionali sono medio-grandi, appartengono in misura maggiore al settore delle macchine per l'industria e hanno formalizzato più delle altre aziende la propria innovazione attraverso la brevettazione. In misura minore è invece la percentuale delle imprese di questa categoria che ritiene che i rapporti con le aziende locali giovino alla capacità competitiva dell'azienda.

Tab. 3: Differenze fra categorie di imprese competitive

\begin{tabular}{|l|c|c|c|}
\hline & $\begin{array}{c}\text { Imprese } \\
\text { competitive a livello } \\
\text { locale o nazionale } \\
\text { (percentuale di } \\
\text { imprese) }\end{array}$ & $\begin{array}{c}\text { Imprese competitive a } \\
\text { livello internazionale } \\
\text { (percentuale di } \\
\text { imprese) }\end{array}$ & $\begin{array}{c}\text { Pearson's } \\
\text { chi- } \\
\text { squared } \\
\text { test }\left(\chi^{2}\right)\end{array}$ \\
\hline Dimensione ( $\geq 50$ dipendenti) & $16,67 \%$ & $46,15 \%$ & $*$ \\
\hline Settore macchine per l'industria & $44,44 \%$ & $84,62 \%$ & $* *$ \\
\hline Dipartimento R\&S & $55,56 \%$ & $53,85 \%$ & \\
\hline Background HR studi tecnici/ingegneristici & $94.44 \%$ & $92.31 \%$ & \\
\hline Brevetti & $22.22 \%$ & $53.85 \%$ & $*$ \\
\hline Innovazione all'avanguardia & $22.22 \%$ & $23.08 \%$ & \\
\hline Innovazioni di marketing e/o organizzative & $50.00 \%$ & $53.85 \%$ & \\
\hline Rilevanza sistema di imprese locali & $70.59 \%$ & $56.67 \%$ & \\
\hline Collaborazioni internazionali & $27.78 \%$ & $7.69 \%$ & \\
\hline Sviluppo sui mercati esteri: & & & $*$ \\
\hline $\begin{array}{l}\text { Vendita sui mercati globali (Inclusivi di altri } \\
\text { paesi oltre che Europa e Nord America) }\end{array}$ & $66.67 \%$ & $100 \%$ & $*$ \\
\hline $\begin{array}{l}\text { Miglior strategia di accesso ai mercati esteri: } \\
\text { nuovi prodotti e/o nuovi servizi }\end{array}$ & $22.22 \%$ & $53.85 \%$ & \\
\hline
\end{tabular}

${ }^{*}$ P value significativo al $5 \% ;{ }^{*} \mathrm{P}$ value significativo al $10 \%$

Fonte: elaborazioni proprie sui dati del questionario.

Una più attenta osservazione sul tipo di sviluppo che le imprese hanno effettuato sui mercati esteri negli ultimi 3 anni fa emergere come le aziende più competitive sono riuscite ad espandere la propria attività di export a livello globale. Tali aziende dimostrano di aver intrapreso in misura maggiore rispetto alle altre (e con maggior successo) strategie di accesso ai mercati esteri attraverso l'introduzione di nuovi prodotti e/o servizi piuttosto che attraverso la vendita di prodotti/servizi in cui premiante risulta solo la qualità del prodotto o la convenienza di prezzo. Per una buona percentuale di queste aziende gli indici di performance economiche risultano cresciuti negli ultimi anni più che per le altre (graf. 1). 
Graf. 1: Percentuale di imprese con performance economiche in aumento negli ultimi 3 anni per tipologia di imprese competitive

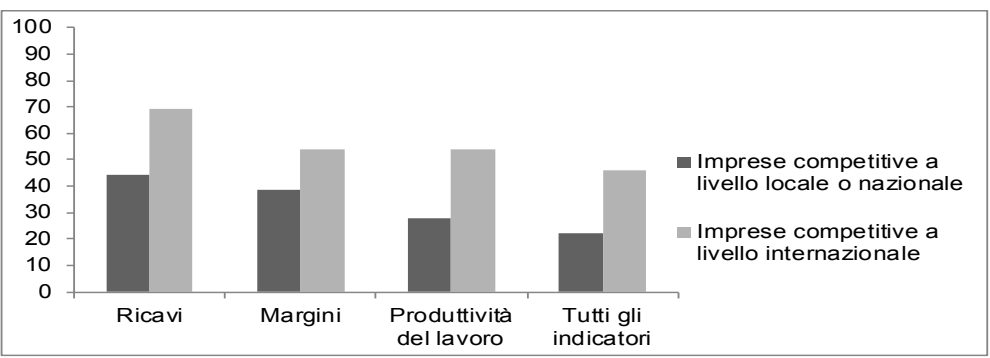

Fonte: elaborazioni proprie sui dati del questionario. Per produttività del lavoro si intende rapporto tra valore aggiunto e dipendenti

Le performance economiche tratte dai dati di bilancio AIDA danno ulteriormente conferma di alcuni trend emergenti dall'analisi delle risposte al questionario (graf. 2).

\section{Graf. 2: Performance economiche per tipologia di imprese competitive tratte dai dati AIDA}

Ricavi e valore aggiunto pro-capite medio per il triennio 2014-2016 (In Euro)
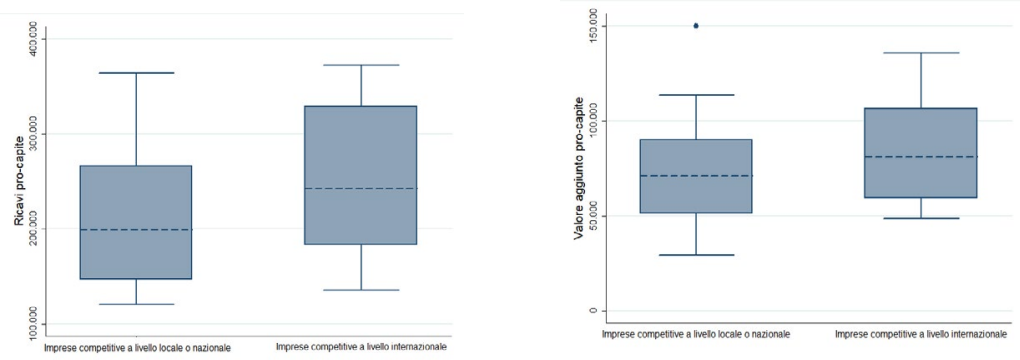

Tasso di variazione dei ricavi e valore aggiunto pro-capite medi del triennio 2014-2016 rispetto al triennio 2011-2013
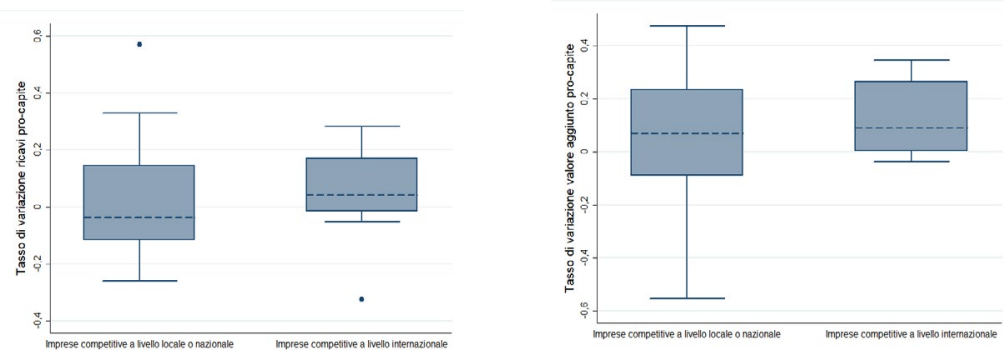

Fonte: elaborazioni proprie su dati AIDA. Nei box plot il limite inferiore dei quadranti grigi rappresenta il $1^{\circ}$ quartile dei valori relativi all'indicatore, mentre il limite superiore del quadrante il $3^{\circ}$ quartile. La linea tratteggiata al centro rappresenta la mediana, mentre le linee orizzontali alle estremità della linea verticale, sia in alto che in basso, rappresentano rispettivamente i valori di minimo e massimo della distribuzione. I punti isolati sono da considerarsi come outlier. Per 3 imprese del campione non è stato possibile analizzare i dati in quanto non presenti su AIDA. 
sinergie Vol. 36, N. 107, 2018

I box plot relativi ai ricavi e valore aggiunto medi pro-capite del triennio 2014-2016 mostrano in generale un miglior andamento del gruppo di imprese a destra ossia di quelle che dopo la crisi hanno dichiarato di essere diventate più competitive a livello internazionale. Lanalisi dei tassi di variazione di tali indicatori rispetto al triennio precedente mostra poi, che anche se una maggior variabilità è presente nel primo gruppo di imprese (imprese divenute più competitive solo a livello locale o nazionale), in media le imprese del secondo gruppo mostrano tassi di variazione dei ricavi e del valore aggiunto più positivi.

\section{Conclusioni}

Il presente lavoro si basa su dati originali raccolti nel settore della meccatronica vicentina nei primi mesi del 2017. Nel rispondere alle domande di ricerca vengono proposte alcune riflessioni sulla capacità competitiva delle imprese del sistema distrettuale e sul grado di competitività raggiunto dalle imprese. Attraverso unanalisi empirica su dati propri raccolti nel 2017 ed integrata con unanalisi di performance economiche tratte dall'analisi dei dati AIDA vengono valutati sia i fattori associati ad un diverso grado di capacità competitiva che le performance competitive raggiunte. Lanalisi suggerisce che per sostenere una capacità competitiva spendibile non è sufficiente investire negli aspetti relativi all’organizzazione e alla commercializzazione - come molte delle imprese del campione stanno facendo - ma si deve andare oltre. Affinché tale capacità permetta alle aziende di competere sui mercati anche internazionali è necessario investire in risorse umane da usare nelle attività innovative. Tali risorse sono necessarie per interfacciare l'azienda con la complessità relazionale $\mathrm{e}$ tecnologica che la globalizzazione e la digitalizzazione stanno innescando.

Oltre a ciò, ulteriori fattori diventano essenziali per accrescere la capacità competitiva. Da una parte, vi è il fondamentale ruolo della capacità di saper produrre beni e servizi con un alto grado di 'novelty', soprattutto legati alla produzione e alla customizzazione del processo. Tale innovazione, formalizzata attraverso un'attività di brevettazione, aumenta la probabilità di raggiungere una capacità competitiva solida sui mercati globali e può favorire l'innesco di un circolo virtuoso fra innovazione tecnologica e competitività. Dall'altra parte, conta il mantenimento di rapporti di rete non solo con le altre aziende del distretto, ma anche con imprese e organizzazioni internazionali con cui l'azienda sviluppa rapporti di collaborazione, rapporti che devono basarsi su un vero e proprio scambio di conoscenze, e non solo sulla semplice acquisizione di fattori o attività.

Se la leadership sui mercati internazionali sembra essere ancora una prerogativa delle imprese di media e grande dimensione, il presente lavoro dimostra che un certo livello di competitività può essere raggiunto anche dalle imprese più piccole se riescono a rimanere ancorate ai circuiti virtuosi locali e allo stesso tempo allacciarsi alla rete globale. Il modello di business che la piccola impresa deve perseguire è quello della rete estesa: utilizzare le proprie specifiche risorse competitive ma aumentando la propria capacità di governance dei processi di generazione e circolazione della conoscenza, 
e la propria powership nei rapporti di rete (Rullani, 2010). Diversamente dalle imprese che mantengono una capacità competitiva geograficamente più circoscritta, le aziende che hanno dichiarato di sentirsi addirittura più forti della concorrenza internazionale sembrano perseguire modelli di business sia più formalizzati dal punto di vista innovativo, che con un minore coinvolgimento delle aziende locali rispetto alle altre. L'eterogeneità dei comportamenti che recentemente si evidenzia nei distretti sembra riguardare più quest'ultime imprese che sembrano muoversi in modo più svincolato dal territorio.

Sebbene non sia un argomento trattato in questo paper, l'analisi ulteriore dei dati e le interviste effettuate suggeriscono che la promozione e la governance da parte del territorio sono insufficienti per sostenere la capacità competitiva. Probabilmente il mancato rinnovo del patto distrettuale riguardante la meccatronica a livello regionale ha limitato la promozione di azioni coordinate di sostegno allo sviluppo del sistema. Le imprese devono sapersi inserire sempre più nelle reti globali di innovazione in maniera autonoma. Se il territorio rimane immobile, sono le imprese capaci di lavorare su reti aperte ad aprire i circuiti locali al nuovo che avanza.

Dal punto di vista manageriale le implicazioni del presente lavoro sottolineano la necessità delle imprese di dotarsi di manager con una mentalità aperta, con affermate esperienze estere e capaci di cogliere le opportunità di collaborazione che nascono nei circuiti di rete estesa sia con le organizzazioni che con nuovo capitale umano capace di gestire l'integrazione fra conoscenze tradizionali e nuove. Manager che devono anche essere in grado di individuare nel locale le sacche di eccellenza di fornitura di beni complementari e servizi high-tech con cui crescere insieme.

La politica industriale locale dovrebbe partire dall'incentivazione a questi meccanismi virtuosi di apertura e collaborazione. Il governo locale dovrebbe poi aumentare il grado di integrazione e complementarietà delle proprie politiche con le politiche che supportano l'innovazione e la competitività delle imprese costruite su altri livelli (nazionale, internazionale) per lavorare anchesso in modo specializzato in una rete multilivello di interventi (Bellandi e Caloffi, 2016). Il lavoro sottolinea importanti aspetti della competitività post crisi del sistema distrettuale della meccatronica.

Seppure l'analisi quantitativa sia basata su un numero limitato di imprese, il campione è certamente rappresentativo della popolazione delle aziende, almeno per i sotto-settori qui studiati. Il tipo di variabili utilizzate, il periodo considerato così come il modello proposto non permettono di dare una direzione certa alla causalità delle relazioni e di evitare possibili problemi di endogenità. Sebbene le interviste aiutino a confermare i risultati, future ricerche dovrebbero essere indirizzate ad integrare i dati ottenuti con dati statistici oggettivi relativi agli anni seguenti l'indagine, in modo da cogliere in maniera più adeguata sia le possibili determinanti della capacità competitiva dell'impresa, sia la relazione di quest'ultima con le performance aziendali.
Monica Plechero

capacità competitiva delle imprese del distretto della meccatronica vicentina 


\section{sinergie}

Vol. 36, N. 107, 2018

\section{Bibliografia}

ANTONELLI C. (1995), Economia dell'innovazione: Cambiamento tecnologico e dinamica industriale, Università Laterza.

BARZOTTO M., CORÒ G., VOLPE M. (2016), "Territorial capital as a company intangible”, Journal of Intellectual Capital, vol. 17, n.1, pp. 148-167.

BAILEY D., BELLANDI M., CALOFFI A., DE PROPRIS L. (2010). “Place-renewing leadership: trajectories of change for mature manufacturing regions in Europe", Policy Studies, vol. 31, n. 4, pp. 457-474.

BATHELT H. A., MALMBERG A., MASKELL P. (2004), "Clusters and knowledge: local buzz, global pipelines and the process of knowledge creation”, Progress in Human Geography, vol. 28, n. 1, pp. 31-56.

BECATTINI G. (1998), Distretti industriali e made in Italy, Bollati Boringhieri, Firenze.

BELL M.R., PAVITT K. (1993), “Technological Accumulation and Industrial Growth: Contrasts between developed and developing countries", Industrial and Corporate Change, vol. 2, n. 2, pp. 157- 210.

BELLANDI M., DE PROPRIS L., SANTINI E. (2018). "An Evolutionary Analysis of Industrial Districts: The Changing Multiplicity of Production KnowHow Nuclei", Cambridge Journal of Economics, Online first. https://doi. org/10.1093/cje/bey012.

BELLANDI M., CALOFFI A. (2016), "Industrial policies in a Marshallian-based multilevel perspective”, European Planning Studies, vol. 24, n. 4, pp. 687-703.

BELUSSI F.E., SEDITA S.R. (2009), "Life Cycle vs. Multiple Path Dependency in Industrial Districts”, European Planning Studies, vol. 17, n. 4, pp 505-528.

BELUSSI F.E., SEDITA S.R. (2012), "Industrial Districts as Open Learning Systems: Combining Emergent and Deliberate Knowledge Structures", Regional Studies, vol. 46, n. 2, 165-184.

BOSCHMA R., TER WAL A L.J. (2007), "Knowledge Networks and Innovative Performance in an Industrial District: The Case of a Footwear District in the South of Italy", Industry and Innovation, vol. 14, n. 2, pp. 177-199.

CAINELLI G., EVANGELISTA R., SAVONA M. (2006), "Innovation and economic performance in services: a firm-level analysis", Cambridge Journal of Economics, vol. 30, pp. 435-458.

CASTELLANI D., RULLANI E., ZANFEI A., (2017), "Districts, multinationals and global/digital networks", Economia e Politica Industriale, vol. 44, n. 4, pp. 429-447.

CHAMINADE C., PLECHERO M. (2015), “Do Regions Make a Difference? Regional Innovation Systems and Global Innovation Networks in the ICT Industry", European Planning Studies, vol. 23, n. 2, pp. 215-237.

CHIARVESIO M., DI MARIA E., MICELLI S. (2010), "Global Value Chains and Open Networks: The Case of Italian Industrial Districts”, European Planning Studies, vol. 18, n.3, pp 333-350.

COHEN W.M., LEVINTHAL D.A. (1990), “Absorptive Capacity: A new Perspective on Learning and Innovation", Administrative Science Quarterly, vol. 35, $\mathrm{n}$. 1, pp. 128-152.

COLOMBO M.G., GRILLI L. (2005), "'Founders' Human Capital and the Growth of New Technology-Based Firms: A Competence-Based View”, Research Policy, vol. 34, n.6, pp. 795-816. 
COLTORTI F., (2015), "Le medie imprese del settore meccanico elettronico", in Unioncamere, Osservatorio nazionale distretti italiani. Il nuovo respiro dei distretti tra ripresa e Riposizionamento, Rapporto 2015, pp.105-111.

COLTORTI F., RESCINITI R., TUNISINI A., VARALDO R. (Eds.), (2012), Midsized Manufacturing Companies: The New Driver of Italian Competitiveness, Springer, Milano.

DE MARCHI V., DI MARIA E., GEREFFI G. (eds), (2018), Local Clusters in Global Value Chains. Linking Actors and Territories Through Manufacturing and Innovation, Routledge, Abingdon.

DEI OTTATI G. (2009), "An industrial district facing the challenges of globalization: Prato today", European Planning Studies, vol. 17, n. 1, pp. 1817-35.

FARACE S., MAZZOTTA F. (2015), "The effect of human capital and networks on knowledge and innovation in SMEs", Journal of Innovation Economics and Management, vol. 1, n. 16, pp. 39-71.

GUSTAVSSON P., HANSSON P., LUNDBERG L. (1999), “Technology, resource endowments and international competitiveness", European Economic Review, vol. 43, n. 8, pp. 1501-1530.

HALL B.H., LOTTI F., MAIRESSE J. (2009), "Innovation and productivity in SMEs: empirical evidence for Italy", Small Business Economics, vol. 33, n. 1, pp. 13-33.

INTESA SAN PAOLO, (2015), "Economia e finanza dei distretti industriali", Direzione Studi e Ricerche, Rapporto annuale, n. 8.

ITAMI H. (1987), Mobilizing Invisible Assets, Harvard University Press, Boston.

MALERBA F. (2005), "Sectoral systems of Innovation: a framework for linking innovation to the knowledge base, structure, and dynamics of sectors", Economics of Innovation and New Technology, vol. 14, n. 1-2, pp-63-82.

MARSHALL A. (1920), Principles of Economics (eighth edition), Porcupine Press, Philadelphia.

MASKELL P., MALBERG A. (1999), "Localised Learning and Industrial Competitiveness", Cambridge Journal of Economics, vol. 23, n. 2, pp. 167-86.

MARTIN R., WIIG ASLESEN A., GRILLITSCH M., HERSTAD S.J. (2018), "Regional Innovation Systems and Global Flows of Knowledge", in Isaksen A., Martin R., Trippl M. (eds), New Avenues for Regional Innovation Systems - Theoretical Advances, Empirical Cases and Policy Lessons, Springer, Cham, pp. 127-47.

MEDIOBANCA E UNIONCAMERE (2015), Le medie imprese industriali italiane (2004- 2013), Milano.

MORRISON A. (2008), “Gatekeepers of knowledge' within industrial districts: who they are, how they interact", Regional Studies, vol. 42, n. 6, pp. 817-835.

OECD (2005), Oslo Manual: Guidelines for Collecting and Interpreting Innovation Data, 3rd Edition, OECD Publishing, Paris.

PAVITT K. (1984), "Sectoral Patterns of Technical Change: Towards a Taxonomy and a Theory", Research Policy, vol. 13, n. 6, pp. 343-373.

PENROSE E.G. (1959), The theory of the grow th of the firm, Wiley, UK.

PLECHERO M., RULLANI E. (2007), Innovare. Reinventare il made in Italy, Egea, Milano.

PLECHERO M., CHAMINADE C. (2013), "The Influence of Micro-Characteristics in Different Modes of Globalization of Innovation: A Comparative Study of Indian (Pune) and Chinese (Beijing) Firms", Industry and Innovation, vol. 20, n.7, pp. 661-682.
Monica Plechero

capacità competitiva delle

imprese del distretto della

meccatronica vicentina 
PORTER M. (1990), The Competitive Advantage of Nations, The Free Press, New York, NY.

RABELLOTTI R., CARABELLI A., HIRSCH G. (2009), "Italian Industrial Districts on the Move: Where Are They Going?”, European Planning Studies, vol. 17, n. 1, pp. 19-41.

RICCIARDI A. (2013), "I distretti industriali italiani: recenti tendenze evolutive", Sinergie, n. 91, pp. 21-58.

RULLANI E. (2004), Economia della conoscenza. Creatività e valore nel capitalismo delle reti, Carocci Editore, Milano.

RULLANI E. (2010), Modernità sostenibile. Idee, filiere e servizi per uscire dalla crisi, Marsilio Editori, Venezia.

RULLANI E. (2015), "Distretti e filiere in evoluzione", in Unioncamere (2015) Osservatorio nazionale distretti italiani. Il nuovo respiro dei distretti tra ripresa e Riposizionamento, Rapporto 2015, pp.76-101.

SEDITA S.R., DE NONI I., PILOTTI L. (2017), "Out of the crisis: an empirical investigation of place-specific determinants of economic resilience", European Planning Studies, vol. 25, n. 2, pp. 155-180.

TEECE D.J. (1980), "Economies of scope and the scope of the enterprise", Journal of Economic Behavior and Organization, vol. 1, n. 3, pp. 223-247.

UNIONCAMERE (2014), Osservatorio nazionale distretti italiani. Idee, esperienze e progetti per rafforzare o ricostruire la competitività dei territori.

UNIONCAMERE (2015), Osservatorio nazionale distretti italiani. Il nuovo respiro dei distretti tra ripresa e Riposizionamento.

UNIVERSITAS MERCATORUM (2013), Il modello di organizzazione e gestione della sicurezza semplificato per le aziende del distretto veneto della meccatronica, Universitas Mercatorum, Roma.

VARALDO R. (2014), La nuova partita dell'innovazione. Il futuro dell'industria in Italia, Il Mulino, Bologna.

WERNEFELT B. (1984), "A resource-based view of the firm", Strategic Management Journal, vol. 5, n. 10, pp. 171-180.

WILLIAMS R. (2006), “Generalized ordered logit/partial proportional odds models for ordinal dependent variables”, Stata Journal, vol. 6, n. 1, pp. 58-82. 
Appendice

Tab. A: Statistica descrittiva e correlazione fra le principali variabili
Monica Plechero

Fuori dalla crisi: la

capacità competitiva delle imprese del distretto della meccatronica vicentina

\begin{tabular}{|c|c|c|c|c|c|c|}
\hline Variabile & Descrizione & $\mathrm{N}$ & Media & $\begin{array}{l}\text { Deviazione } \\
\text { standard }\end{array}$ & Min & Max \\
\hline $\begin{array}{l}\text { Posizione } \\
\text { competitive post } \\
\text { crisi (1) }\end{array}$ & $\begin{array}{l}\text { variabile ordinale: valore } 0 \text { se posizione } \\
\text { debole o stabile; } 1 \text { se posizione più } \\
\text { strategica e selettiva; } 2 \text { se posizione più } \\
\text { competitiva. }\end{array}$ & 84 & 1,142857 & 0,7467658 & 0 & 2 \\
\hline Dimensione (2) & $\begin{array}{l}\text { valore } 1 \text { se dimensione aziendale } \geq 50 \\
\text { dipendenti; } 0 \text { se }<50\end{array}$ & 84 & 0,2261905 & 0,4208766 & 0 & 1 \\
\hline $\begin{array}{l}\text { Settore macchine } \\
\text { industria (3) }\end{array}$ & $\begin{array}{l}\text { valore } 1 \text { se appartenenza al sotto- } \\
\text { settore delle macchine per l'industria; } \\
0 \text { se automazione oppure macchine ed } \\
\text { apparecchi elettrici ed elettronici o altro }\end{array}$ & 84 & 0,4761905 & 0,5024324 & 0 & 1 \\
\hline $\begin{array}{l}\text { Dipartimento } \\
\text { R\&S (4) }\end{array}$ & $\begin{array}{l}\text { valore } 1 \text { se presenza in azienda di un } \\
\text { dipartimento di ricerca e sviluppo, } 0 \\
\text { altrimenti. }\end{array}$ & 84 & 0,3928571 & 0,4913188 & 0 & 1 \\
\hline $\begin{array}{l}\text { Background HR } \\
\text { studi tecnici/ } \\
\text { ingegneristici (5) }\end{array}$ & $\begin{array}{l}\text { valore } 1 \text { se presenza in azienda di } \\
\text { dipendenti impegnati in attività innovativa } \\
\text { con almeno un profilo di studi tecnici e di } \\
\text { ingegneria, } 0 \text { altrimenti. }\end{array}$ & 84 & 0,7857143 & 0,4127903 & 0 & 1 \\
\hline Brevetti (6) & $\begin{array}{l}\text { valore } 1 \text { se deposito di brevetti da parte } \\
\text { dell'azienda, } 0 \text { altrimenti }\end{array}$ & 84 & 0,2619048 & 0,4423118 & 0 & 1 \\
\hline $\begin{array}{l}\text { Innovazione } \\
\text { all'avanguardia } \\
\text { (7) }\end{array}$ & $\begin{array}{l}\text { valore } 1 \text { se è stata introdotta un'innovazione } \\
\text { all’avanguardia (fra i primi o pochissimi a } \\
\text { livello mondiale), } 0 \text { altrimenti. }\end{array}$ & 84 & 0,1309524 & 0,3393744 & 0 & 1 \\
\hline $\begin{array}{l}\text { Innovazioni di } \\
\text { marketing e/o } \\
\text { organizzative (8) }\end{array}$ & $\begin{array}{l}\text { valore } 1 \text { se sono state introdotte innovazioni } \\
\text { di marketing oppure organizzative, } 0 \\
\text { altrimenti. }\end{array}$ & 84 & 0,452381 & 0,5007166 & 0 & 1 \\
\hline $\begin{array}{l}\text { Rilevanza sistema } \\
\text { di imprese locali } \\
(9)\end{array}$ & $\begin{array}{l}\text { valore } 1 \text { se è stata attribuita un'importanza } \\
(3 \text { o } 4 \text { su una scala di } 5) \text { alle imprese } \\
\text { locali nel sostenere la capacità innovativa } \\
\text { dell'aziende, } 0 \text { altrimenti. }\end{array}$ & 84 & 0,3452381 & 0,4783014 & 0 & 1 \\
\hline $\begin{array}{l}\text { Collaborazioni } \\
\text { internazionali } \\
\text { (10) }\end{array}$ & $\begin{array}{l}\text { valore } 1 \text { se cè stata collaborazione con altre } \\
\text { imprese/organizzazioni internazionali per } \\
\text { attività di innovazione o produzione in } \\
\text { cui vi sia stato un vero e proprio scambio } \\
\text { di conoscenze (cioè non limitate ad una } \\
\text { semplice acquisizione di fattori o attività); } \\
0 \text { altrimenti. }\end{array}$ & 84 & 0,1309524 & 0,3393744 & 0 & 1 \\
\hline
\end{tabular}

\begin{tabular}{|l|l|l|l|l|l|l|l|l|l|l|}
\hline Correlazioni & $(1)$ & $(2)$ & $(3)$ & $(4)$ & $(5)$ & $(6)$ & $(7)$ & $(8)$ & $(9)$ & $(10)$ \\
\hline$(1)$ & - & & & & & & & & & \\
\hline$(2)$ & 0.1260 & - & & & & & & & & \\
\hline$(3)$ & $0.2661^{*}$ & 0.0543 & - & & & & & & & \\
\hline$(4)$ & 0.1736 & $0.3808^{*}$ & -0.0349 & - & & & & & & \\
\hline$(5)$ & $0.2178^{*}$ & 0.2130 & -0.1411 & $0.3607^{*}$ & - & & & & & \\
\hline$(6)$ & 0.1772 & $0.2604^{*}$ & 0.0826 & $0.4633^{*}$ & $0.2451^{*}$ & - & & & & \\
\hline$(7)$ & 0.1630 & 0.0432 & -0.0875 & 0.1213 & 0.2027 & $0.2503^{*}$ & - & & & \\
\hline$(8)$ & 0.2117 & 0.1947 & -0.0046 & 0.1504 & 0.0666 & 0.1658 & $0.2853^{*}$ & - & & \\
\hline$(9)$ & $0.3325^{*}$ & -0.0933 & -0.1910 & -0.0201 & 0.0131 & -0.0908 & 0.0150 & -0.0060 & - & \\
\hline$(10)$ & 0.0679 & 0.0432 & 0.0538 & 0.1213 & 0.1167 & 0.1701 & 0.1631 & -0.0692 & -0.0592 & - \\
\hline
\end{tabular}

* $\mathrm{P}$ value significativo al 5\%. Tutte le variabili dipendenti sono variabili dummy. Se non specificato diversamente le attività sono relative agli ultimi 3 anni (2014-2016).

sinergie italian journal of managemen

ISSN 0393-5108 DOI $10.7433 / \mathrm{s} 107.2018 .07$

pp. 139-157

\section{Monica Plechero}

Research Fellow of Applied economics

University of Florence - Italy

e-mail: monica.plechero@unifi.it 